\title{
Cognitive Vertical Handover in Heterogeneous Networks
}

\author{
Yu-Jui Liu and Shin-Ming Cheng \\ Department of Computer Science and Information Engineering, \\ National Taiwan University of Science and Technology, \\ Taipei 106, Taiwan. \\ \{M10215078, smcheng\}@mail.ntust.edu.tw
}

\author{
Po-Yao Huang \\ School of Computer Science, \\ Carnegie Mellon University, \\ PA 15213, USA. \\ poyaoh@cs.cmu.edu
}

\begin{abstract}
One of the major design issues in heterogeneous wireless networks is the support of seamless vertical handover for mobile users. The cognitive capability for mobile terminal enables the functionalities of system parameter estimation and learning. By utilizing these parameters, cognitive users make a handover decision to select an appropriate point of attachment (PoA) among multiple primary radio access technologies/networks without explicit negotiation with surrounding PoAs, and thus handover latency and signaling overhead are minimized. A novel cognitive vertical handover process is proposed including system capacity estimation mechanisms and vertical handover decision process. The vertical handover decision problem is formulated as a Markov decision process and its performance is better than other distributed schemes which do not consider the network side's information from the numerical results.
\end{abstract}

Index Terms-cognitive radio, heterogeneous networks, markov decision process, vertical handover

\section{INTRODUCTION}

The next generation networks are expected to exhibit heterogeneity of Radio Access Technologies (RATs) consisting of various wireless networks (e.g., WiMAX and WiFi) and cellular communications (e.g., WCDMA, HSPA and 5G). These heterogeneous RATs with different data rates, sizes of coverage and access costs are integrated together to complement their different characteristics and provide mobile users the ability of universal access. In addition, mobility issue in transport network, Distributed Mobility Management (DMM) [1] is a general solution for wireless network to address the problem. Thus, mobile user should receive services even as it moves across different RATs with service continuity and Quality of Service (QoS) guarantees.

To provide seamless mobility, mobile terminals (MTs) are envisioned to be equipped with multiple RAT interfaces to establish connection with one of points of attachment (PoAs) based on the communication environment and the application requirements. The operation of switching from one RAT to another is defined as vertical handover [2]. The subject of enabling vertical handover between heterogeneous RATs while optimizing session continuity has received considerable attention, where MT is suggested to gain knowledge about all heterogeneous RATs in the area of interest to facilitate vertical handovers. For example, MT should know the available bandwidth of surrounding PoAs to prevent the connection after han- dover being dropped due to resource deficiency. Traditionally, the information is obtained through the negotiation between MT and PoAs. Thus, what kinds of RAT measurements and information should be exchanged and how to provide the necessary interactions are the most important considerations to achieve seamless vertical handover.

Regarding these issues, a vertical handover scheme based on QoS [3] which considers Received Signal Strength (RSS) and Bandwidth is proposed to provide a unified way for optimizing handover decisions. What's more, we also consider the power consumption [4] and signaling cost as important factors to affect decisions. However, the negotiation between MT and PoAs increases handover latency and degrades the performance. Cognitive radio (CR) [5], [6] appears as a different way of promising solution where a cognitive MT is capable of dynamically sensing unused spectrum segments in a target spectrum pool, and communicating via the unused spectrum segments without causing harmful interference to the primary users, where the primary users are defined as those of existing RATs. Thus, MT can detect the availability of PoAs of different RATs and select the most appropriate one according to the gathering information without negotiation between PoA and thus performance of handover is enhanced.

Due to the enhanced features, we design a cognitive vertical handover process involving the following phases: (i) initialization; (ii) sensing and reasoning; (iii) decision making; and (iv) handover execution. When the received signal strength (RSS) from the serving PoA is under an acceptable threshold, vertical handover will be initialized. During the sensing phase, physical layer waveforms are sensed to determine the existence of each RAT. What's more, MT further estimates system parameters before practically connecting to PoA through some RATs. According the measured parameters, the MT determines whether the connections should continue the existing RAT or be switched to another one in the decision making phase. Finally, in the handover execution phase, the MT reconfigures its physical layer and connections are rerouted from the existing PoA to selected one in a seamless and secured manner in the network side.

In this paper, we detail the unique features of how a $\operatorname{cog}$ nitive MT measures and estimates RAT-specific information to facilitate vertical handover decision with QoS guarantee. 
This paper adopts a decision-theoretic approach by casting the design of handover decision in the framework of Markov Decision Process (MDP) [7]. We associate a reward function with the QoS received by the connection to candidate PoA and cost function with the signaling overhead and processing load incurred when vertical handover execution is performed, respectively. The objective is to determine one of the candidate PoAs that maximizes the expected summation of reward and cost. Since the candidate PoAs only include three PoAs with stronger RRS and thus the complexity of optimal solution by adopting MDP is acceptable. The contributions of this paper are listed as follows.

- MT measures and learns system parameters by spectrum sensing without the assistance of network side, which leads reduction of handover latency.

- A MDP located in MT is proposed to select the best one of surrounding PoAs according to the observed information.

The remainder of this paper is organized as follows: Section II summarizes the existing literatures on vertical handover in heterogeneous networks. Section III gives an overview of the system model consisting of network model and operations of vertical handover. Section IV concentrates on the system parameters estimation at MT. The proposed MDP-based decision algorithm is presented in Section V. Section VI conducts intensive simulations to evaluate the performance of proposed algorithm. Finally, we conclude this work with contributions in Section VII.

\section{RELATED WORK}

Obviously, the handover decision process needs to take both RSS information from MT and status of resource in PoA into consideration to avoid choosing a PoA having available resource but with poor signal quality or having strong RSS but with no free channel [8]. Following presents the existing literatures considering both mobile and network information for vertical handover in heterogeneous networks, which are classified according to handover decision strategy [9].

Mobile-Assisted Handover (MAHO) is the strategy that MT measures the signaling of surrounding PoAs and the network employs this information and decides whether or not to trigger handover. The vertical handover decision process becomes an element of Common Radio Resource Management (CRRM), which also includes admission control and rate control. In [2], Zhu and McNair first presented cost functions at network side for handover decision considering available bandwidth and RSS of the available RATs. With optimization, this algorithm significantly improves the system performance in terms of throughput and blocking probability [10]. New metrics for vertical handover decision continue to emerge in the following literatures, such as revenue [11], load balance among PoAs [12], and battery lifetime of MT [13].

Network-Assisted Handover (NAHO) is the strategy opposing to MAHO. In this case, network side supports some

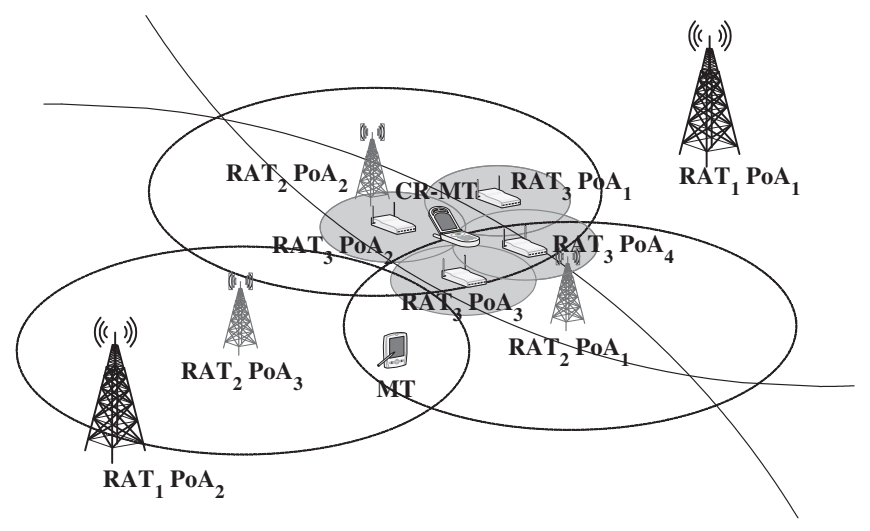

Fig. 1. Network Architecture

system parameters to MT actively for vertical handover decision. Since the computing ability of MT is limited, optimal decision may not be feasible and thus the amount of information offered by network should be carefully considered to prevent significantly negotiation delay caused by the excess of information.

Except the MT-monitored RSS and load of surrounding PoAs [14], several parameters are taken into account for handover decision, such as user preference [15], RAT access cost [16], QoS parameters of surrounding PoAs [17], or operator policies [18]. However, in these works, no technical details were provided on how MT obtains (or network provides) network side information.

It is worth noting that NAHO is a suitable solution for MT without spectrum sensing capacity [19]. However, the further assistance from the networks may not necessary for MT since it can retrieve system parameters through spectrum sensing, which makes the case that MT is solely responsible for making decisions possible.

\section{SYSTEM MODEL}

\section{A. Network Architecture}

We consider an integrated wireless and mobile system having $M$ different types of primary RAT networks as shown in Fig. 1. Each network is divided into many individual coverage areas (CAs), and each CA has one PoA. Suppose that PoAs of a specific RAT are homogeneous, that is, all PoAs belong to primary $\operatorname{RAT}_{i}(1 \leq i \leq M)$ have same capacity $L_{i}$, which is defined as the number of logic channels.

The MT is assumed to be capable to reconfigure to $M$ RATs and is assumed to record the existences of at most $N$ PoAs (index by $j$ ) with best RRS for each primary RAT. For example, in Fig. 1, MT can detect the existence of $\mathrm{PoA}_{1}$ and $\mathrm{PoA}_{2}$ of RAT $1, \mathrm{PoA}_{1}$ and $\mathrm{PoA}_{2}$ of $\mathrm{RAT}_{2}$, and $\mathrm{PoA}_{1}$ to $\mathrm{PoA}_{4}$ of $\mathrm{RAT}_{3}$. Note if $N=3$, MT only selects three of PoAs of $\mathrm{RAT}_{3}$ with better RRS as handover candidates and records their specific information such as available resource. When the MT is moving, the RSS or specific information from surrounding PoAs changes and thus handover may occur. 


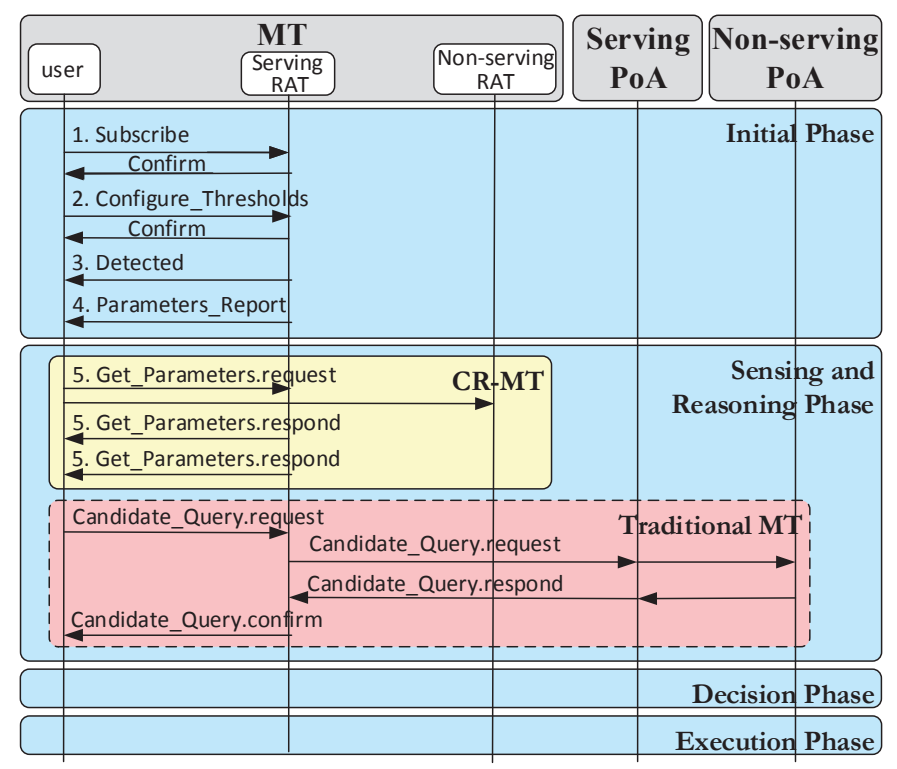

Fig. 2. Message flow of Cognitive Vertical Handover

To prevent the interference caused by opportunistic channel access from MT, we assume PoAs of primary RAT $j$ admits or rejects requests from MT according to the same admission control policy where guard channel policy [8] is adopted. In particular, when the number of channels allocated for PoA of RAT $j$ exceeds the threshold $T_{j}$, the request from MT is rejected. And we assume the MT knows the policy and threshold.

\section{B. Cognitive Vertical Handover Procedure}

Fig. 2 clearly represents the mobile-initiated handover procedure at MT. In this figure, the operations of vertical handover is divided into four phases: initiation, sensing and reasoning, decision, and execution phases. In the initiation phase, the MT subscribes events for the link parameters report on the serving RAT (1). In particular, MT configures threshold on the serving RAT to report radio measurements when specific thresholds are crossed (2). The type of this measurement report may indicate an urgent handover request or just a periodic informational message. Then the MT may detect one of surrounding PoA through (3) and adds it into handover candidates.

When MT receives the first link indication (4) reporting that the RSS of serving RAT is below thresholds setting in (2), it enters the sensing and reasoning phase to obtain and estimate the system parameters through every RAT (5). On the other hand, legacy-MT only obtains the RAT-specific information by querying serving and candidate PoAs, which cause significant delay. By using information retrieved from spectrum sensing or supported by PoAs, MT periodically makes a vertical handover decision. In the execution phase, the MT may updates location information or establishes security associations with a target PoA while mobility signaling communication and radio path redirection are executed in the network side.

\section{System CAPACITY Estimation IN VARIOUS RATS}

This section discusses the spectrum sensing functionality at MT from different kinds of RATs, where spectrum typically is divided into a set of multiple orthogonal logical channels using frequency division multiple access (FDMA), time division multiple access (TDMA), code division multiple access (CDMA), or antenna and its polarization state of multipleinput multiple-output (MIMO).

In recent years, spectrum sensing techniques [6] have been widely discussed to detect and identify the existence of primary systems. Energy detection, CP detection, pilot detection and spatiotemporal sensing, etc, are some of the mechanisms to determine the instantaneous spectrum availability of specific channel. At time $t_{s}$ (time index), the MT may access the channel for its own use or communicate with the primary system if the channel is idle (available). Define $1_{k}(s)$ as the indicator function denoting the channel availability of $k$ th channel at time $t_{s}$ :

$$
1_{k}(s)= \begin{cases}1, & \text { channel } k \text { is available at }\left[t_{s}, t_{s+1}\right), \\ 0, & \text { otherwise. }\end{cases}
$$

With the help of spectrum sensing mechanisms that identify instantaneous channel availabilities, the number of active users in multiple access systems can be estimated. [20] utilizes energy detection for TDMA system identification. Regarding to FDMA, systems activities are determined by multiple signal classification [21]. Number of active users in the CDMA system is discussed in [22]. For the most proceeding communication technique OFDMA, hybrid methods with a decision tree structure is proposed in [23]. The generalized results of these algorithms using Eq. (1) are as follows. In TDMA systems, the channel availabilities are represented as:

$$
\mathbf{1}(s)=\left\{1_{k}(s), \ldots, 1_{k}(s+t), \ldots, 1_{k}(s+T-1)\right\},
$$

where $T$ is the gathered period for load determination algorithm (in the following part). In FDMA systems, the channel availabilities are

$$
\mathbf{1}(s)=\left\{1_{0}(s), \ldots, 1_{k}(s), \ldots, 1_{K-1}(s)\right\}^{\prime},
$$

where $K$ is the number of frequency bands. Note that here the orthogonal channels are not limited to time and frequency bands. Code, pattern, or spatial domain methods are possible choices and thus the notation is extendable for various systems. The generalized form of channel availability matrix of is defined as

$$
\begin{aligned}
\mathbf{1}(s) & =\left\{1_{k}(s+t)\right\} \\
& =\left(\begin{array}{ccc}
1_{0}(s) & \cdots & 1_{0}(s+T-1) \\
\vdots & \ddots & \vdots \\
1_{K-1}(s) & \cdots & 1_{K-1}(s+T-1)
\end{array}\right),
\end{aligned}
$$

where total number of channels is $K \times T$. Let $g(\mathbf{1}(s))$ denotes the load-determination function which maps the spectrum sensing results to the system load. In this paper, we define the system load as the number of channel occupied and propose a 
simple load-determination function. The system load $l$ of PS can be intuitively determined by:

$$
l=g(\mathbf{1}(s))=g\left(\sum_{k=0}^{K-1} \sum_{t=0}^{T-1} 1_{k}(s+t)\right) .
$$

For a MT with total sensing capability, the load $l_{i, j}$ of $i$ th PoA of RAT $j$ can be derived. Note that if only $i-1$ surrounding PoAs of RAT $j$ can be detected by MT, $l_{i, j}$ is set as 0 . The resulting load estimation in the MT is:

$$
\mathbf{L}=\left\{l_{i, j}\right\}=\left(\begin{array}{ccc}
l_{1,1} & \cdots & l_{1, N} \\
\vdots & \ddots & \vdots \\
l_{M, 1} & \cdots & l_{M, N}
\end{array}\right)
$$

The estimated load information is utilized and served in our MDP for handover and system selection.

\section{Model of Markov Decision Process}

\section{A. State Space}

The states in our MDP consist of current PoA choice and current PoA loading (calculated from environment information). We define current PoA connection indicator as:

$$
\mathbf{B}=\left\{I D_{i j} \cdot r_{i j}: i=1, \ldots, N, j=1, \ldots, M\right\},
$$

where $I D_{i j}$ and $r_{i j}$ respectively represents the identity and RSS of the $i$-th PoA that MT perceives through RAT $j . I D_{i j}$ value is -1 if this PoA is connected by MT currently and is 1 else. Among the detected available PoAs, MT records first $N$ PoAs with largest RSS. The current PoA loading is determined by the MT through (6). Thus, we define the state space of MT as follows.

$$
\mathbf{S}=\{\mathbf{B}, \mathbf{L}\},
$$

where $\mathbf{B}, \mathbf{L}$ are $N \times M$ matrix. For example, for the MT choosing the second PoA of the third RAT among all PoAs sensed, it's state may be:

$$
\mathbf{S}=\{\underbrace{\left(\begin{array}{ccc}
r_{11} & r_{12} & r_{13} \\
r_{21} & 0 & -r_{23} \\
0 & 0 & r_{33}
\end{array}\right)}_{\text {Indicator }}, \underbrace{\left(\begin{array}{ccc}
l_{11} & l_{12} & l_{13} \\
l_{21} & 0 & l_{23} \\
0 & 0 & l_{33}
\end{array}\right)}_{\text {PoA loading }}\} .
$$

Note that in this example MT only senses two surrounding PoAs of the RAT 1 and one PoAs of RAT 2. Then the state will be further sent into a reward function. Note that the key idea is to compact the vector form into matrix form, they are same for MDP.

\section{B. Decision Epochs}

We use the sequence $T=\{1, \ldots, K\}$ represents the times of successive decision epochs, where the random number $K$ denotes the time that connection terminates and is assumed to be geometrically distributed with mean $\frac{1}{(1-\lambda)}$. Then

$$
\operatorname{Pr}[K=k]=\lambda^{k-1}(1-\lambda) .
$$

\section{Action Space}

At each decision epoch, an action is chosen that determines how the admission control will perform at the next decision moment. We denote the action set as

$$
A=\left\{a_{i j}: i=1, \ldots, N, j=1, \ldots, M\right\},
$$

where $a_{i j}$ is binary indicators, each of which has a value of 1 if and only if the MT handovers to $i$ th PoA with RAT $j$.

\section{State Transition Probabilities}

Given that the current state is $\mathbf{s}=\left(b_{i j}, l_{i j}: i=\right.$ $1, \ldots, N, j=1, \ldots, M)$ and the chosen action is $a_{p q} \in \mathbf{A}$, the state transition probability function for the next state $\mathbf{s}^{\prime}=\left(b_{i j}^{\prime}, l_{i j}^{\prime}: i=1, \ldots, N, j=1, \ldots, M\right)$ is determined by

$\operatorname{Pr}\left(\mathbf{s}^{\prime} \mid \mathbf{s}, a\right)= \begin{cases}\prod_{i=1}^{N} \prod_{j=1}^{M} \operatorname{Pr}\left(b_{i j}^{\prime}, l_{i j}^{\prime} \mid b_{i j}, l_{i j}\right), & a_{p q}=1, \\ 0, & a_{p q}=0\end{cases}$

This function is Markovian because the state transition depends on the current state and action but not on the previous states. It is reasonable to assume $b_{i j}$ are independent of $l_{i j}$.

\section{E. Decision Rule}

A decision rule prescribes a procedure for action selection in each state at a specified decision epoch. Deterministic Markovian decision rules are functions: $\delta_{t}: \mathbf{S} \rightarrow \mathbf{A}$, which specifies the action choice when the system occupies state $\mathrm{s}$ at decision epoch $t$. Here we simply assume a stationary policy $\pi$ which represents that a fixed decision rule is used at all decision epochs.

\section{F. Rewards Function}

Given the current state $s \in \mathbf{S}$ and the chosen action $a \in \mathbf{A}$, the reward function $r(s, a)$ is defined as $c(s, a)-p(s, a)-$ $d(s, a)$, where $c(s, a), p(s, a)$, and $d(s, a)$ denote capacity reward function, power consumption function, and delay cost function, respectively. The capacity reward function $c(s, a)$ is defined as follows.

$$
c(s, a)= \begin{cases}-\infty, & \text { if } r_{p q}<\theta_{q}, \\ g\left(r_{p q}, l_{p q}\right), & \text { if } r_{p q} \geq \theta_{q} .\end{cases}
$$

Where $g(s, a)$ denote the throughput reward function of $p$ th POA and $q$ th RAT with $r_{p q}$ (RSS) and $l_{p q}$ (load). The power consumption function $p(s, a)$ is defined as $p(s, a)=p_{p q}$. The signaling cost function $\mathrm{d}(\mathrm{s}, \mathrm{a})$ is defined as follows.

$$
d(s, a)= \begin{cases}c_{i j}, & \text { if } j \neq q, \\ c_{j}, & \text { if } j=q \text { and } i \neq p, \\ 0, & \text { if } j=q \text { and } i=p .\end{cases}
$$

Given such an MDP, a decision problem is to choose an optimal action at every decision epoch to maximize the expected value of the total reward accrued during the connection's lifetime, which is defined as

$$
v^{\pi}(s)=E_{s}^{\pi}\left[\sum_{t=1}^{\infty} \lambda^{t-1} r\left(s_{t}, a_{t}\right)\right],
$$



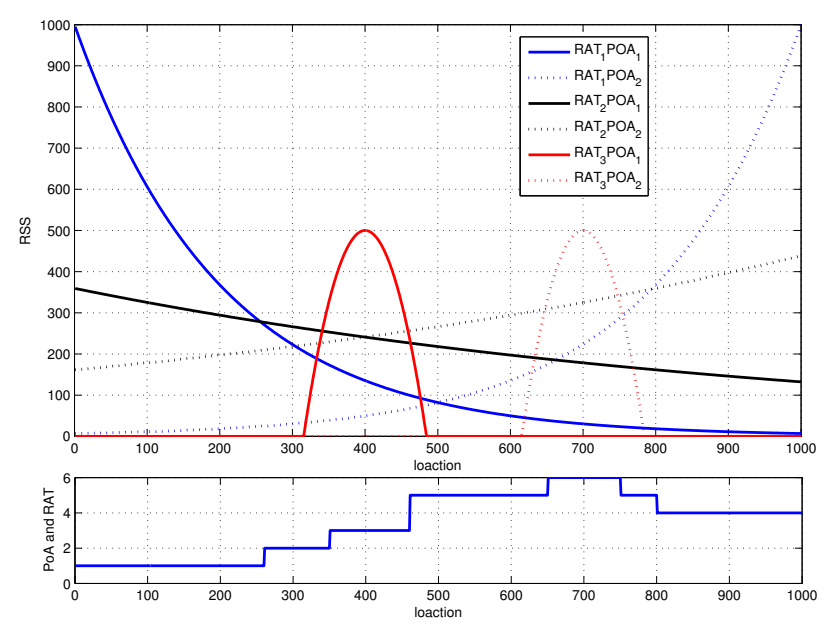

Fig. 3. The selection results of MT in heterogeneous network

where a policy $\pi=\left(\delta_{1}, \delta_{2}, \ldots, \delta_{K}\right)$ is a sequence of decision rules to be used at all decision epochs. An optimal policy for such a problem is to act greedily with respect to the optimal value function, defined recursively the optimality equations:

$$
\begin{aligned}
v(s) & =\max _{a \in A} v^{\pi}(s) \\
& =\max _{a \in A}\left\{r(s, a)+\sum_{s^{\prime} \in S} \gamma \operatorname{Pr}\left(s^{\prime} \mid s, a\right) v\left(s^{\prime}, t+1\right)\right\} .
\end{aligned}
$$

This paper adopts policy iteration algorithm (PIA) [7] to solve (16), where a stationary deterministic optimal policy and the corresponding expected total reward are determined.

\section{Simulation AND Numerical Results}

It is obvious that the proposed cognitive vertical handover process in MT outperforms the exiting ones in MT without cognitive capability in terms of signaling delay and overhead. As shown in Fig. 2, the negotiations between PoA and MT are omitted and thus signaling delay and overhead can be minimized. Thus, the performance of the MDP optimal policy $\delta^{\mathrm{M}}$ for $\mathrm{MT}$ is compared with the case where traditional MT selects one of surrounding PoAs only considering RSS (denoted as $\delta^{\mathrm{R}}$ ). This implies that MT does not retrieve any RAT-specific information through explicit negotiation and thus it has the same signaling overhead as that for MT. We also consider the policy where the PoA with the highest available bandwidth will be selected for comparison and we denote this policy as $\delta^{\mathrm{B}}$. The performance metrics are the expected total reward per connection, which is defined in (15). The time between successive decision epochs is assumed to be seconds.

The simulation parameters are summarized in I, we assume three RATs collocated (i.e., $M=3$ ) and MT at most can record status of two PoAs of each RAT (i.e., $N=2$ ). The figure on the top of Fig. 3 shows the RSS received by MT in different location of collocated RATs network. The capacity of three RATs are 100, 50, and 20 and initial values of loading in each PoAs of each RATs is 50, 20 (RAT 1), 15, 12 (RAT

\begin{tabular}{|c|c|c|c|}
\hline Symbols & RAT1 & RAT2 & RAT3 \\
\hline$\gamma$ & 0.9 & 0.9 & 0.9 \\
\hline$C_{i j}$ & 5 unit time & 10 unit time & 15 unit time \\
\hline$P_{p q}$ & $5 \mathrm{~W}$ & $10 \mathrm{~W}$ & $15 \mathrm{~W}$ \\
\hline
\end{tabular}

TABLE I

Simulation PARAMETERS

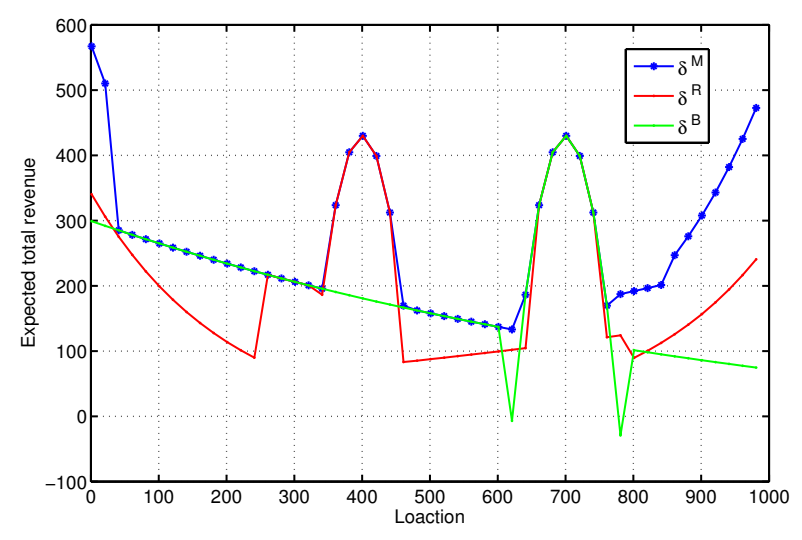

Fig. 4. Expected total revenue reward for three policies

2), 10 and 8 (RAT 3). For simplicity, the switching costs are the same. The lower figure of Fig. 3 shows the selection result of MDP policy.

Fig. 4 plots the expected total revenue of three policies against MT's location in the same simulation environment. Note that $\delta^{\mathrm{R}}$ implies the traditional MT only considering RSS for decision making without the aid of network side. Under the similar signaling overhead and delay, MT with MDP policy outperforms traditional MT with $\delta^{\mathrm{R}}$. Obviously, $\delta^{\mathrm{M}}$ has better performance than that of $\delta^{\mathrm{B}}$.

\section{CONCLUSION}

In this paper, we propose an cognitive vertical handover process for MT in heterogeneous networks. By applying the cognitive functionality, MT can sense and estimate the loading of surrounding PoAs of various RATs directly without the aid of network side. As a resulting, it is significant to reduce handover latency and signaling overhead. Next, mechanisms to measure and understand the loading of surrounding PoAs of various RATs are proposed. According to the information such as loading and RSS from surrounding PoAs, we propose a MDP-based handover decision process for MT where both network access cost and power consumption are also considered. Numerical results show that our MDP-based algorithm outperforms the existing scheme where cognitive capability is not available.

\section{REFERENCES}

[1] F. Giust, L. Cominardi, and C. J. Bernardos, "Vertical handoffs in fourthgeneration multinetwork environments," IEEE Commun. Mag., vol. 53, no. 1, pp. 142-149, Jan. 2015.

[2] J. McNair and Z. Fang, "Vertical handoffs in fourth-generation multinetwork environments," IEEE Wireless Commun. Mag., vol. 11, no. 3, pp. 8-15, Jun. 2004. 
[3] A. Ahmed, L. M. Boulahis, and D. Gaiti, "Enabling vertical handover decisions in heterogeneous wireless networks: A state-of-the-art and a classification," IEEE Commun. Surveys Tuts., vol. 16, no. 2, pp. 776809, Apr. 2014.

[4] S. M. I. Yujae Song, Peng-Yong Kong and I. Youngnam Han, Senior Member, "Power-optimized vertical handover scheme for heterogeneous wireless networks," IEEE Commun. Lett., vol. 18, no. 2, pp. 277-280, Feb. 2014.

[5] J. Mitola and G. M. Jr., "Cognitive radio: Making software radios more personal," IEEE Personal Commun. Mag., vol. 6, no. 4, pp. 13-18, Aug. 1999.

[6] S. Haykin, "Cognitive radio: Brain-empowered wireless communications," IEEE J. Sel. Areas Commun., vol. 23, no. 2, pp. 201-210, Feb. 2005.

[7] M. L. Puterman, Markov Decision Processes: Discrete Stochastic Dynamic Programming. Wiley-Interscience, 2005.

[8] B. B. Madan, S. Dharmaraja, and K. S. Trivedi, "Combined guard channel and mobile-assisted handoff for cellular networks," IEEE Trans. Veh. Technol., vol. 57, no. 1, pp. 502-510, Jan. 2008.

[9] K. Pahlavan, P. Krishnamurthy, and A. Hatami, "Handoff in hybrid mobile data networks," IEEE Personal Commun. Mag., vol. 7, no. 2, pp. 34-47, Apr. 2000

[10] L. Satori, S.-E. Elayoubi, B. Fourestié, and Z. Nouir, "On the WiMAX and HSDPA coexistence," in Proc. IEEE ICC 2007, Jun. 2007, pp. 56365641.

[11] F. Yu and V. Krishnamurthy, "Optimal joint session admission control in integrated WLAN and CDMA cellular networks with veritical handoff," IEEE Trans. Mobile Comput., vol. 6, no. 1, pp. 126-139, Jan. 2007.

[12] W. Shen and Q.-A. Zeng, "Cost-function-based network selection strategy in integrated wireless and mobile networks," IEEE Trans. Veh. Technol., vol. 57, no. 6, pp. 3778-3788, Nov. 2008.

[13] S. Lee, K. Sriram, K. Kim, Y. H. Kim, and N. Golmie, "Vertical handoff decision algorithms for providing optimized performance in heterogeneous wireless networks," IEEE Trans. Veh. Technol., vol. 58, no. 2, pp. 865-881, Feb. 2009.

[14] K. Yang, I. Gondal, and B. Qiu, "Multi-dimensional adaptive SINR based vertical handoff for heterogeneous wireless networks," IEEE Commun. Lett., vol. 12, no. 6, pp. 438-440, Jun. 2008.

[15] Q. Song and A. Jamalipour, "Network selection in an integrated wireless LAN and UMTS environment using mathematical modeling and computing techniques," IEEE Wireless Commun. Mag., vol. 12, no. 3, pp. 42-48, Jun. 2005.

[16] C. Sun, E. Stevens-Navarro, and V. W. S. Wong, "An MDP-based vertical handoff decision algorithm for heterogeneous wireless networks," IEEE Trans. Veh. Technol., vol. 57, no. 2, pp. 1243-1254, Mar. 2008.

[17] C. Makaya and S. Pierre, "An architecture for seamless mobility support in IP-based next-generation wireless networks," IEEE Trans. Veh. Technol., vol. 57, no. 2, pp. 1209-1225, Mar. 2008.

[18] P. Chiron, E. Njedjou, P. Seite, K. Gosse, E. Melin, and P. Roux, "Architectures for IP-based network-assisted mobility management across heterogeneous networks," IEEE Wireless Commun. Mag., vol. 15, no. 2, pp. 18-25, Apr. 2008.

[19] M. Liu, Z. Li, X. Guo, and E. Dutkiewicz, "Performance analysis and optimization of handoff algorithms in heterogeneous wireless networks," IEEE Trans. Mobile Comput., vol. 7, no. 7, pp. 846-857, Jul. 2008.

[20] G. Ganesan and Y. Li, "Cooperative spectrum sensing in cognitive radio networks," in Proc. IEEE DySPAN 2005, Nov. 2005, pp. 137-143.

[21] C. K. Yu and K. C. Chen, "Multiple systems sensing for cognitive radio networks over rayleigh fading channel," in Proc. IEEE VTC Spring 2008, May 2008, pp. 1574-1578.

[22] W. C. Wu and K. C. Chen, "Identification of active users in synchronous CDMA multiuser detection," vol. 16, no. 12, pp. 1723-1735, Dec. 1998.

[23] S. Y. Tu, K. C. Chen, and R. Prasad, "Spectrum sensing of OFDMA systems for cognitive radio networks," IEEE Trans. Veh. Technol., vol. 58, no. 7, pp. 3410-3425, Feb. 2009. 